\title{
An iterative statistical tolerance analysis procedure to deal with linearized behavior models*
}

\author{
Antoine DUMAS ${ }^{\dagger 1,2}$, Jean-Yves DANTAN ${ }^{1}$, Nicolas GAYTON ${ }^{2}$, Thomas BLES ${ }^{1}$, Robin LOEBL ${ }^{1}$ \\ ( ${ }^{1}$ Laboratoire Conception Fabrication Commande, Arts et Métiers ParisTech Metz, METZ CEDEX 3 57078, France) \\ ( ${ }^{2}$ Institut Pascal, Clermont Université, IFMA, UMR 6602, BP 10448, Clermont-Ferrand 63000, France) \\ †E-mail: antoine.dumas@ensam.eu
}

Received July 25, 2014; Revision accepted Oct. 19, 2014; Crosschecked Apr. 13, 2015

\begin{abstract}
Tolerance analysis consists of analyzing the impact of variations on the mechanism behavior due to the manufacturing process. The goal is to predict its quality level at the design stage. The technique involves computing probabilities of failure of the mechanism in a mass production process. The various analysis methods have to consider the component's variations as random variables and the worst configuration of gaps for over-constrained systems. This consideration varies in function by the type of mechanism behavior and is realized by an optimization scheme combined with a Monte Carlo simulation. To simplify the optimization step, it is necessary to linearize the mechanism behavior into several parts. This study aims at analyzing the impact of the linearization strategy on the probability of failure estimation; a highly over-constrained mechanism with two pins and five cotters is used as an illustration for this study. The purpose is to strike a balance among model error caused by the linearization, computing time, and result accuracy. In addition, an iterative procedure is proposed for the assembly requirement to provide accurate results without using the entire Monte Carlo simulation.
\end{abstract}

Key words: Tolerance analysis, Probability of failure, Linearization of behavior model, Optimization, Monte Carlo simulation

doi:10.1631/jzus.A1400221

Document code: A

CLC number: TH161

\section{Introduction}

Due to the imprecision associated with the manufacturing process, it is not possible to attain the theoretical dimensions associated with the process in a repetitive manner. It may cause a degradation of the product performance. To ensure the desired behavior and the performance of the system in spite of manufacturing imprecisions, the component characteristics are assigned a tolerance zone within which the value of the characteristics, i.e., situation and intrinsic, lie.

Moreover, technology is always increasing and performance requirements continually tighten, and

\footnotetext{
* Project supported by the French National Research Agency (No. ANR-11-MONU-013)

(7) ORCID: Antoine DUMAS, http://orcid.org/0000-0002-7414896X

(C)Zhejiang University and Springer-Verlag Berlin Heidelberg 2015
}

also the costs and the required precision of assembly operations increase. More attention is needed in tolerance design to be able to manufacture highprecision assemblies at lower costs. Therefore, tolerance analysis is a key element in the manufacturing industry to improve product quality and to decrease the manufacturing costs (Qureshi et al., 2012).

Tolerance analysis concerns the verification of functional requirements after tolerances have been specified on each isolated part. Analysis methods are divided into two categories (Dantan and Qureshi, 2009):

1. Displacement accumulation whose goal is to model the influences of the deviations on the geometrical behavior of the mechanism. This relationship uses the following form: $Y=f(\boldsymbol{X}, \boldsymbol{G})$ (Nigam and Turner, 1995), where $Y$ is the response of the system (a characteristic such as a gap or a functional 
characteristic), $\boldsymbol{X}$ is the vector of deviation values (situation or/and intrinsic deviations) of parts composing the mechanism, and $G$ is the vector of gaps between the parts of the mechanism. The function $f$ represents the deviation accumulation of the mechanism; it can be an explicit analytical expression, an implicit analytical expression, or a numerical simulation. The difficulty in determining the function $f$ increases with the complexity of the studied system (Mhenni et al., 2007; Ballu et al., 2008). In the case of analytical formulation, the mathematical formulation of the behavior model can be written from topological loop relations of the system as well as constraints to prevent surfaces from penetrating into others. In addition, the analysis method must consider the worst admissible configuration of gaps which is realized using an optimization algorithm. However, non-linear analytical models are difficult to handle in an optimization problem. Indeed, Qureshi et al. (2012) noticed that some optimization results were not admissible because constraints were violated. Non-linear optimization algorithms may not find the global minimum which is required to consider the worst case scenario. In addition, using genetic evolutionary algorithms might be a solution, but these algorithms are very time-consuming and it will be irrelevant to use within numerical simulations, such as Monte Carlo (MC) simulations. This is why a linearization of non-linear equations is required.

2. Tolerance accumulation aims at simulating the composition of tolerances (i.e., linear tolerance accumulations, 3D accumulations) (Ameta et al., 2011). The admissible deviations are mapped using several vector spaces in a region of hypothetical parametric space. The relevant literature mentions several techniques to represent geometrical tolerances or dimensioning tolerances, among which are T-maps (Davidson et al., 2002; Bhide et al., 2005; Davidson and Shah, 2012), gap spaces (Zou and Morse, 2003; Morse, 2004), and deviation domains (Giordano and Duret, 1993; Giordano et al., 2005). All these methods require mathematical tools, such as the Minkowski sums and the intersection of domains (Mansuy et al., 2011), so as to compute the quality level of the product. To be able to perform these operations, behavior models must be in a linear form.

Both categories need a linearization of the behavior model to compute the quality levels of the product. In fact, tolerance analysis is totally depen- dent on the models chosen to describe the system behavior.

This study focuses on the impact of linearization of the behavior model on the statistical tolerance analysis which is based on the displacement accumulation theory. In particular, the impact of the strategy of linearization is studied. To manage the probability of out-tolerance products and evaluate the impact of component tolerances on product performance, designers need to simulate the influences of manufacturing imprecisions with respect to the functional requirements. In this case, the goal of tolerance analysis is to predict a quality level during the design stage, the technique consists of computing two probabilities of failure, one relative to the assembly $P_{\mathrm{fa}}$ and one relative to the functionality $P_{\mathrm{f}}$.

This paper intends to show that the linearization procedure has an impact on the probability of failure accuracy and presents an adaptive algorithm able to compute accurate probability faster than the classical solution method.

\section{Statistical tolerance analysis of over- constrained mechanisms}

We can distinguish three main issues in tolerance analysis (Dantan et al., 2012; Qureshi et al., 2012):

1. The models representing the geometrical deviations;

2. A mathematical model of the geometrical behavior with deviations;

3 . The development of the analysis methods.

This study focuses on the impact of the second issue on the third issue. The goal of this section is to describe the current method to solve a tolerance analysis problem, from the formulation to a solution method. Section 2.1 deals with the mathematical formulation of a statistical tolerance analysis problem. Section 2.2 shows the analysis method used to compute the probabilities of failure.

\subsection{Formulation of a tolerance analysis problem}

Most mechanisms have gaps between parts which make the behavior model more complex to be modeled. Gaps are considered as free variables, they depend on the geometrical deviations and on 
the parts configuration of the mechanism (Qureshi et al., 2012). The behavior model must take into account those gaps by limiting their displacement to avoid the interpenetration of one surface into another. Boundaries are therefore defined using interface constraints written as

$$
\left\{C_{\mathrm{i}}^{(k)}(\boldsymbol{X}, \boldsymbol{G}) \leq 0\right\}_{k=1,2, \ldots, N_{C_{\mathrm{i}}}},
$$

where $N_{C_{\mathrm{i}}}$ is the number of interface constraints. The mechanical behavior of the mechanism is modeled using compatibility equations, these are the composition relations of displacements in the various topological loops. The set of equality equations provides a linear system to be satisfied. They are written as

$$
\left\{C_{\mathrm{c}}^{(k)}(\boldsymbol{X}, \boldsymbol{G})=0\right\}_{k=1,2, \ldots, N_{C_{\mathrm{c}}}},
$$

where $N_{C_{\mathrm{c}}}$ is the number of compatibility equations.

The formulation of both requirements is based on quantifiers. The assembly requirement is given by Qureshi et al. (2012):"For all admissible deviations, there exists a gap configuration such as the assembly requirements and the behavior constraints are verified". The assembly probability of failure $P_{\mathrm{fa}}$ is then given in Eq. (3). The technique to compute this probability is to minimize one interface constraint subject to all compatibility equations and interface constraints to be satisfied. A solution means that the assembly is possible, on the contrary the assembly is impossible if no solution exists.

$$
\begin{aligned}
& P_{\mathrm{fa}}=1- \\
& \operatorname{Prob}\left(\exists \boldsymbol{G} \in \mathbb{R}^{m} \mid C_{\mathrm{i}}(\boldsymbol{X}, \boldsymbol{G}) \leq 0 \bigcap C_{\mathrm{c}}(\boldsymbol{X}, \boldsymbol{G})=0\right) .
\end{aligned}
$$

The functional requirement is also defined by Qureshi et al. (2012) :"For all admissible gap configurations, the geometrical behavior and the functional requirements are verified". The functional requirement implies that the functional characteristic $Y$ is not to exceed a threshold value $Y_{\mathrm{th}}$. A functional condition is defined, $C_{\mathrm{f}}=Y_{\mathrm{th}}-Y \geq 0$, a positive value ensures the mechanism is functional. However, all configurations do not have to be checked, indeed, to compute the functional probability $P_{\mathrm{f}}$ of failure, it is necessary to find at least one admissible configuration where the functional condition is not respected:

$$
P_{\mathrm{f}}=\operatorname{Prob}\left(\exists \boldsymbol{G}_{\mathrm{adm}} \in \mathbb{R}^{m} \mid C_{\mathrm{f}}(\boldsymbol{X}, \boldsymbol{G}) \leq 0\right),
$$

where $\boldsymbol{G}_{\mathrm{adm}}$ are gap values verifying all interface constraints and compatibility equations. This corresponds to the worst gap configuration to be found; this configuration provides the worst value of the functional condition. The technique to find the worst admissible configuration of gaps for the functional condition is to minimize $C_{\mathrm{f}}$ subject to all constraints, as shown in Eq. (5).

$$
\begin{aligned}
& P_{\mathrm{f}}=\operatorname{Prob}\left(\min _{\boldsymbol{G}} C_{\mathrm{f}}(\boldsymbol{X}, \boldsymbol{G}) \leq 0\right), \\
& \text { with } C_{\mathrm{c}}(\boldsymbol{X}, \boldsymbol{G})=0, C_{\mathrm{i}}(\boldsymbol{X}, \boldsymbol{G}) \leq 0 .
\end{aligned}
$$

\subsection{Solution method based on Monte Carlo simulation and optimization}

The classic solution method combines a Monte Carlo simulation and an optimization algorithm (Dantan and Qureshi, 2009; Qureshi et al., 2012). The functional condition is linear. In addition, all constraints are linear, if not, the linearization procedure proposed in Section 3 is applied. An optimization scheme using a simplex technique is therefore chosen to solve the optimization problems. The different steps of the solution procedure are described below:

1. Defining the Monte Carlo population: a set of $N$ samples $\boldsymbol{x}=\left\{x^{(1)}, x^{(2)}, \ldots, x^{(N)}\right\}$ from the random vector $\boldsymbol{X}$ is created;

2. Launching the optimization algorithm for each sample;

3. Estimating $P_{\mathrm{fa}}$ and/or $P_{\mathrm{f}}$, the probabilities of failure are estimated using the following equations:

$$
\begin{gathered}
\tilde{P}_{\mathrm{fa}}=\frac{1}{N} \sum_{i=1}^{N} I_{\mathrm{D}_{\mathrm{fa}}}\left(x^{(i)}\right), \\
\tilde{P}_{\mathrm{f}}=\frac{1}{N} \sum_{i=1}^{N} I_{\mathrm{D}_{\mathrm{f}}}\left(x^{(i)}\right),
\end{gathered}
$$

where $I_{\mathrm{D}}(\boldsymbol{X})$ is the indicator function; for the assembly requirement the function is:

$$
I_{\mathrm{D}_{\mathrm{fa}}}(\boldsymbol{X})= \begin{cases}1, & \text { no solution can be found } \\ 0, & \text { a solution is found }\end{cases}
$$

For the functional requirement, it is defined as

$$
I_{\mathrm{D}_{\mathrm{f}}}(\boldsymbol{X})= \begin{cases}1, & C_{\mathrm{f}}\left(\boldsymbol{X}, \boldsymbol{G}_{\min }\right) \leq 0 \\ 0, & C_{\mathrm{f}}\left(\boldsymbol{X}, \boldsymbol{G}_{\min }\right)>0\end{cases}
$$


The number of samples $N$ can be defined to yield a coefficient of variation, see Eq. (10), lower than a given accuracy, usually lower than at least $10 \%$.

$$
\operatorname{COV}_{P_{\mathrm{f}}}=\sqrt{\frac{1-P_{\mathrm{f}}}{N P_{\mathrm{f}}}}
$$

\section{Illustration of the linearization procedure}

This section shows the different considered linearization strategies. Section 3.1 presents a simple mechanism to show what type of non linear equations needs to be linearized. Section 3.2 describes the mathematical linearization procedure on such non linear constraints.

\subsection{Illustration on 2D mechanism}

The example in Fig. 1 is a simplified version in 2D of the industrial mechanism shown in Fig. 6 . This mechanism is constituted of two pins, part (1), in relative displacement with part (2). Both pins are fixed in a workpiece. Geometrical deviations are considered in this mechanism (Qureshi et al., 2012):

1. Intrinsic deviations: diameters of pins and their pin-holes: $d_{1 a}, d_{2 a}, d_{1 b}$, and $d_{2 b}$.

2. Situation deviations: orientation and position variations of the substitute surfaces $a$ and $b$ with respect to their respective nominal surfaces. The deviation of the substitute surface $a$ of part (1) with respect to its nominal surface is written $u_{1 a 1}$ and $v_{1 a 1}$ (translation along the $x$-axis and $y$-axis) and $\gamma_{1 a 1}$ (rotation around the $z$-axis).

Small displacement torsors (Bourdet and Clément, 1976; Legoff et al., 2004) are used to represent gaps between the joints of the mechanism. Two torsors are built to model variations in orientation and location of each pin. These torsors are written as $\left\{G_{1 a / 2 a}\right\}_{A}$ and $\left\{G_{1 b / 2 b}\right\}_{B}$ where " $1 a / 2 a, A$ " means
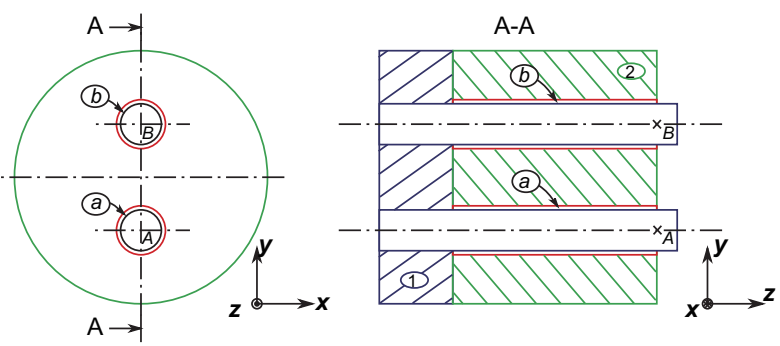

Fig. 1 Representation of the simplified 2D hyperstatic mechanism with a side view these are the variations of the surface $a$ of part (1) with respect to surface $a$ of part (2) applied to point $A$, and expressed as

$$
\left\{G_{1 a / 2 a}\right\}_{A}=\left\{\begin{array}{cc}
- & u_{1 a 2 a A} \\
- & v_{1 a 2 a A} \\
\gamma_{1 a 2 a} & -
\end{array}\right\} .
$$

The geometrical behavior of this mechanis$\mathrm{m}$ is made up of three compatibility equations $C_{\mathrm{c}}(\boldsymbol{X}, \boldsymbol{G})=0$ and two interface constraints $C_{\mathrm{i}}(\boldsymbol{X}, \boldsymbol{G}) \leq 0$ characterizing the noninterpenetration of the pins in their pin-holes. These constraints are given by the quadratic Eqs. (12) and (13).

$$
\begin{aligned}
& C_{\mathrm{i}}^{(1)}=u_{1 a 2 a A}^{2}+v_{1 a 2 a A}^{2}-\left(\frac{d_{2 a}-d_{1 a}}{2}\right)^{2} \leq 0, \\
& C_{\mathrm{i}}^{(2)}=u_{1 b 2 b B}^{2}+v_{1 b 2 b B}^{2}-\left(\frac{d_{2 b}-d_{1 b}}{2}\right)^{2} \leq 0,
\end{aligned}
$$

with $d_{1 a}<d_{2 a}$ and $d_{1 b}<d_{2 b}$.

These inequations specify an admissible displacement area of the center $A$ and $B$ of both pins to not penetrate part (1) (Fig. 2). Linearization has to occur on such constraints to simplify the optimization scheme. Indeed, handling non linear constraints is more difficult than linear constraints. In addition, both Eqs. (12) and (13) are similar, the linearization procedure is therefore illustrated on a reference equation, shown in Eq. (14).

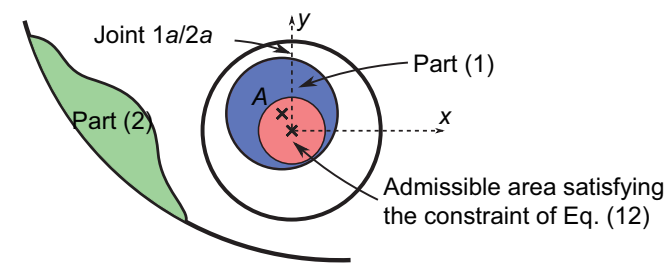

Fig. 2 Admissible area of displacement of pin center $A$ to satisfy the interface constraints of joint $1 a / 2 a$

\subsection{Illustration of linearization strategies on constraints}

This section describes different strategies for the linearization of the non linear equations. These equations result from a cylinder type joint and provide quadratic interface constraints. For a simple 2D circle, the quadratic equation to be linearized is written as

$$
C_{\mathrm{i}}(u, v)=u^{2}+v^{2}-\Delta R^{2},
$$


where $\Delta R$ is the circle radius difference between $u$ and $v$, representing the displacements along the $x$ and $y$-axis.

Linearization corresponds to a discretization of the admissible area of displacement, which is a $2 \mathrm{D}$ circle, into a polygon whose number of facets depends on the number $N_{d}$ of discretizations. Several discretization strategies are considered (Fig. 3). Given $C_{\mathrm{i}}(u, v)=u^{2}+v^{2}-\Delta R^{2}$, an interface constraint, the linearization operation provides new inequations depending on the type of linearization:

Type 1: Discretization following an inner polygon:

$$
C_{\mathrm{i}}^{(k)}(u, v)=u \cos \theta_{k}+v \sin \theta_{k}-\Delta R \cos \frac{\theta_{1}}{2} \leq 0 .
$$

Type 2: Discretization following an outer polygon:

$$
C_{\mathrm{i}}^{(k)}(u, v)=u \cos \theta_{k}+v \sin \theta_{k}-\Delta R \leq 0,
$$

where $\theta_{k}=2 k \pi / N_{d}$, for $k=1,2, \ldots, N_{d}$, is an angle whose parameter $N_{d}$ enables the number of linearizations to be adjusted, and so $\theta_{1}=2 \pi / N_{d}$. One interface constraint becomes $N_{d}$ interface constraints, increasing significantly the number of constraints, but these constraints have the advantage of being linear in displacement.

\section{Confidence interval based algorithm for the assembly requirement}

In this section, an algorithm is proposed to compute an accurate probability faster than the current solution method. The procedure is based on a Monte Carlo simulation and takes advantage of the confidence interval on the probability of failure obtained by combining both linearization strategies for a given number of linearizations. This algorithm is tested

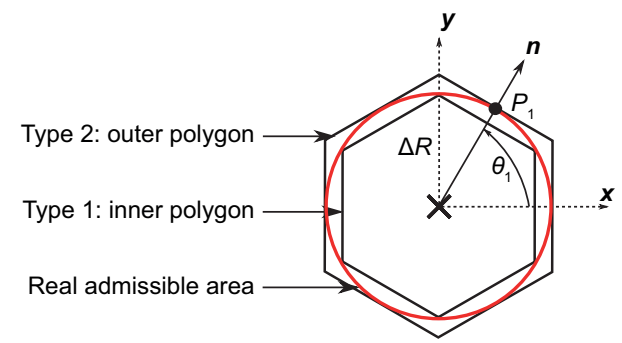

Fig. 3 The two types of discretization of the real admissible area of displacement, here with six facets and compared with the classical Monte Carlo method in Section 5.

The assembly requirement has two interesting properties that are used in the proposed algorithm. First, considering the inner strategy, if a point enables the mechanism to be assembled with a given number of linearizations $N_{d 1}$, then it will always be the case with a greater number of linearizations $N_{d 2}>N_{d 1}$ on the condition that the edges of each polygon coincide (Fig. 4). So to improve the probability accuracy with this strategy, it is sufficien$\mathrm{t}$ to evaluate again, with a greater number of linearizations, only the non-assembly points. As for the second property, for a given number of linearizations $N_{d}$, the entire set of non-assembly points with the outer strategy is included in the set of the nonassembly points with the inner strategy. It means that to compute the probability of failure with the outer strategy, it is sufficient to only evaluate the non-assembly points with the inner strategy.

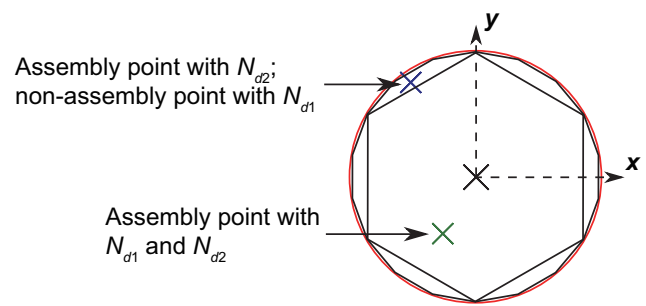

Fig. 4 Two inner polygons with different number of linearizations: $N_{d 1}=6, N_{d 2}=18$

Furthermore, all non-assembly points found with the outer strategy will always be whatever a greater value $N_{d}$. Fig. 5 shows a linearization of a real area of displacement with both strategies, uncertain points can therefore be defined: these are points between the inner and the outer polygons.

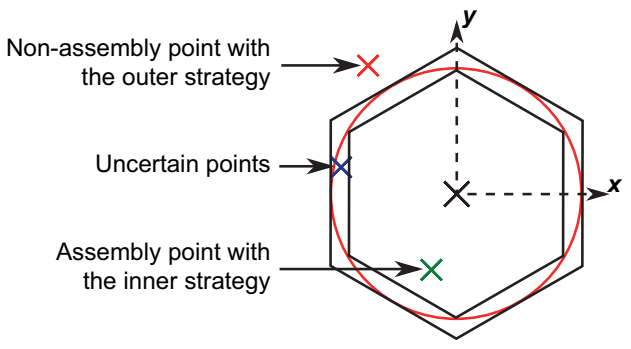

Fig. 5 Two polygons with the inner and outer strategies for the same number of linearizations

The goal of the algorithm is to achieve a classical Monte Carlo simulation with a small number 
of linearizations $N_{d}$. The probability of failure with the inner strategy is first computed. By computing again only non-assembly points from the previous simulation with the outer strategy, the probability of failure for this case can be quickly evaluated. The relative confidence interval, see Eq. (17), between both probabilities can be computed; if this interval is small enough, then the procedure ends, otherwise the number of linearizations is increased and the procedure is launched again only on uncertain points. The algorithm steps are described below:

1. Set a small number of linearizations, e.g., $N_{d 0}=4$.

2. Achieve a Monte Carlo simulation with the inner strategy: compute $P_{\mathrm{fa} \_ \text {inner }}$.

3. Save non-assembly points from the Monte Carlo simulation (points outside the inner polygon).

4. Perform a Monte Carlo with the previous saved points with the outer strategy: compute $P_{\text {fa_outer }}$.

5. Save uncertain points: non-assembly points with the inner strategy and assembly points with the outer strategy (points outside the inner polygon and into the outer polygon).

6. Compute the relative confidence interval (RCI):

$$
\mathrm{RCI}=\frac{P_{\mathrm{fa} \_ \text {inner }}-P_{\mathrm{fa} \_ \text {outer }}}{P_{\mathrm{fa} \_ \text {inner }}} .
$$

7. Compare RCI to a precision criterion, e.g., $5 \%$. If RCI is greater than the criterion, then start the while loop with a greater number of linearizations $N_{d}: N_{d 1}=3 N_{d 0}$.

8. Do while loop using the uncertain points.

(a) Compute $P_{\mathrm{fa}_{-} \text {inner }}$ and save non-assembly points among the uncertain points.

(b) Compute $P_{\text {fa_outer }}$ and update the list of uncertain points.

(c) Compute RCI.

(d) If RCI $<5 \%$, then stop, else $N_{d(k+1)}=$ $3 N_{d k}$.

The RCI is preferred to the confidence interval (CI) because this criterion is independent of the order of magnitude of the probability, so the stopping criterion can correspond to a percentage. This algorithm is tested in the next section for an industrial application.

\section{Estimation of the probability of assembly failure on an industrial mechanism}

The study of the impact and the application of the proposed procedure are applied on a test case based on a Radiall electrical connector (Fig. 6). The mechanism is composed of two parts which must be assembled, and they are positioned by five cotters and two pins which make the mechanism highly overconstrained. The behavior model is not detailed in this paper because of too many equations:

1. Seventy-two compatibility equations;

2. Forty linear interface constraints;

3. Four quadratic interface constraints;

4. Thirty-eight random variables;

5. Seventy-five gap variables.

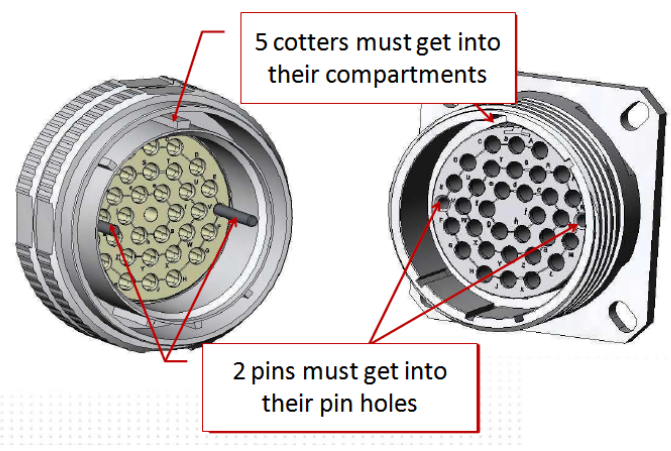

Fig. 6 Illustration of the Radiall connector

All parameter values: dimensions, means, standard deviations, and probability laws, are necessary for tolerance analysis. However, for confidentiality reasons, tolerances and values are changed from the real values for this study. Random variables are defined following a normal distribution. For this test case, only the assembly requirement is evaluated. The probability $P_{\mathrm{fa}}$ will only be computed.

A range of the linearization number is defined and Monte Carlo simulations are performed on each case. The number of samples $N$ is chosen to yield a coefficient of variation on the probability of failure (Eq. (10)) about 6\%.

Results are given in Table 1, which show that the number of linearizations $N_{d}$ and the strategy influence the probability of failure values. Strategies provide different results which converge toward the same value, validating the linearization equations. Considering a target probability to be reached, the 
inner polygon strategy provides conservative results (only valid for the assembly case). In this case, even if the result is an approximation, the real probability value will not be underestimated because this strategy overestimates the probability. Furthermore, the combination of the inner and outer polygon strategies provides a CI for the true failure probability value. The greater the number of linearizations, the smaller the CI.

Table 1 Probabilities of failure values for the assembly requirement obtained with the Monte Carlo simulation and the proposed procedure

\begin{tabular}{lcccccc}
\hline \multirow{2}{*}{$N_{d}$} & \multicolumn{2}{c}{$P_{\mathrm{fa}}\left(\times 10^{-5}\right)$} & \multicolumn{2}{c}{ MC time $(\mathrm{h})$} & Procedure & RCI \\
\cline { 2 - 5 } & Inner & Outer & Inner & Outer & time $(\mathrm{h})$ & $(\%)$ \\
\hline 4 & 185.0 & 22.0 & 8.45 & 8.45 & 8.45 & 88.1 \\
12 & 91.0 & 72.3 & 10.80 & 10.80 & 8.45 & 20.5 \\
36 & 88.7 & 88.3 & 22.60 & 22.60 & 8.45 & 0.4 \\
\hline \multicolumn{7}{c}{$N=3 \times 10^{6} ; \mathrm{COV}=6.1 \% ; 95 \% \mathrm{CI}=2.17 \times 10^{-5}$} \\
\end{tabular}

The computing time increases with the number of linearizations. In addition, it is not possible to know a priori for which the linearization number value for the probability of failure has converged. To avoid doing blinded simulations, it is very important to dispose of a procedure that is able to provide accurate results as quickly as possible. The proposed procedure in Section 4 solves this issue. Indeed, the procedure is an adaptive algorithm which stops when the result is accurate enough whatever the required number of linearizations is to yield a converged result.

This algorithm is performed on the Radiall test case and the results are shown in the bottom part of Table 1. The strategy of linearization (inner or outer) does not influence the computing time for the Monte Carlo simulation, whereas the number of linearizations $N_{d}$ has a greater influence on the computing time and the probability accuracy. Probability values obtained by the proposed procedure are equal to those obtained with the Monte Carlo simulation, because the same sets of samples of the random variables were used. The procedure stopped with a number of linearizations equal to 36 . This procedure ensures that such a number is sufficient to yield a result accurate enough which is not the case with only one Monte Carlo simulation. In addition, the computing time to reach an accurate result is reduced $(8.45 \mathrm{~h})$ compared to the time needed with the classical Monte Carlo simulation (two times $22.6 \mathrm{~h}$ ) for the same accuracy. The proposed procedure computes all values in only one simulation, whereas the Monte Carlo simulation only provides one result at a time.

\section{Conclusions}

The functionality of a product is influenced by design tolerances. Evaluating the quality level of a product at its design stage is therefore a key element; it enables an improvement of the functional quality of the product while reducing the manufacturing cost. This requires methods, such as tolerance analysis, to quantify the impact of tolerances on the mechanism quality. To evaluate the quality level of the product, a mathematical model is required, which must represent its behavior as best as possible. However, the behavior model may be approximated. This approximation provides an inaccurate quality level which is required to be able to manage.

Qureshi et al. (2012) provided a tolerance analysis formulation able to deal with non-linear behaviors. Although the mathematical formulation enables this kind of problem to be solved, a difficulty appears when using an optimization scheme with non-linear constraints, making the result unreliable. The present paper is dedicated to defining linearization strategies for non-linear constraints to solve this problem. It appears that the linearization of non-linear constraints has a real impact on the probability of failure of the mechanism; the obtained result may underestimate the real value, hence over-estimating the quality level. The linearization procedure must be chosen carefully to obtain conservative results. Indeed, depending on the type of requirement (assembly or functional), the conservative strategy is different. In addition, an interesting procedure consists of defining a confidence interval of the true probability of failure using two linearization strategies: outer and inner polygons.

The classical solution method, the Monte Carlo simulation, is not able to provide results for which the chosen number of linearizations is sufficient to yield accurate probabilities of failure. An adaptive procedure is therefore proposed to obtain a confidence interval small enough for the probability. Hence, whatever the studied mechanism, it is possible to obtain a good accuracy for the result, whatever 
the required number of linearizations.

\section{References}

Ameta, G., Serge, S., Giordano, M., 2011. Comparison of spatial math models for tolerance analysis: tolerancemaps, deviation domain, and TTRS. Journal of Computing and Information Science in Engineering, 11(2): 021004. [doi:10.1115/1.3593413]

Ballu, A., Plantec, J.Y., Mathieu, L., 2008. Geometrical reliability of overconstrained mechanisms with gaps. CIRP Annals - Manufacturing Technology, 57(1):159-162. [doi:10.1016/j.cirp.2008.03.038]

Bhide, S., Ameta, G., Davidson, J.K., et al., 2005. Tolerancemaps applied to the straightness and orientation of an axis. Models for Computer Aided Tolerancing in Design and Manufacturing: Selected Conference Papers from the 9th CIRP International Seminar on Computer-Aided Tolerancing, Tempe, Arizona, USA. [doi:10.1007/1-4020-5438-6_6]

Bourdet, P., Clément, A., 1976. Controlling a complex surface with a 3 axis measuring machine. Annals of the CIRP, 25(1):359-361.

Dantan, J.Y., Qureshi, A.J., 2009. Worse case and statistical tolerance analysis based on quantified constraint satisfaction problems and Monte Carlo simulation. Computer-Aided Design, 41(1):1-12. [doi:10.1016/j.cad. 2008.11.003]

Dantan, J.Y., Gayton, N., Dumas, A., et al., 2012. Mathematical issues in mechanical tolerance analysis. 13th Colloque National AIP Priméca, Le Mont-Dore, p.12.

Davidson, J.K., Shah, J.J., 2012. Modeling of geometric variations for line-profiles. Journal of Computing and Information Science in Engineering, 12(4):041004. [doi:10.1115/1.4007404]

Davidson, J.K., Mujezinovic, A., Shah, J.J., 2002. A new mathematical model for geometric tolerances as applied to round faces. Journal of Mechanical Design, 124(4):609-622. [doi:10.1115/1.1497362]

Giordano, M., Duret, D., 1993 . Clearance space and deviation space: application to three-dimensional chain of dimensions and positions. Proceedings of 3rd CIRP Seminar on Computer-Aided Tolerancing, Eyrolles, Paris, p.179-196.

Giordano, M., Samper, S., Petit, J.P., 2005. Tolerance analysis and synthesis by means of deviation domains, axisymmetric cases. Models for Computer Aided Tolerancing in Design and Manufacturing: Selected Conference Papers from the 9th CIRP International Seminar on Computer-Aided Tolerancing, Tempe, Arizona, USA. [doi:10.1007/1-4020-5438-6_10]
Legoff, O., Tichadou, S., Hascoet, J.Y., 2004. Manufacturing errors modelling: two three-dimensional approaches. Proceedings of the Institution of Mechanical Engineers, Part B: Journal of Engineering Manufacture, 218(12):1869-1873. [doi:10.1177/095440540421801219]

Mansuy, M., Giordano, M., Hernandez, P., 2011. A new calculation method for the worst case tolerance analysis and synthesis in stack-type assemblies. Computer-Aided Design, 43(9):1118-1125. [doi:10.1016/j.cad.2011.04. 010]

Mhenni, F., Serré, P., Mlika, A., et al., 2007. Dependency between dimensional deviations in overconstrained mechanisms. Conception et Production Intégrées, 22:4.

Morse, E.P., 2004. Statistical analysis of assemblies having dependent fitting conditions. International Mechanical Engineering Congress and Exposition, p.1-5. [doi:10.1115/IMECE2004-61664]

Nigam, S.D., Turner, J.U., 1995. Review of statistical approaches of tolerance analysis. Computer-Aided Design, 27(1):6-15. [doi:10.1016/0010-4485(95)90748-5]

Qureshi, A.J., Dantan, J.Y., Sabri, V., et al., 2012. A statistical tolerance analysis approach for over-constrained mechanism based on optimization and Monte Carlo simulation. Computer-Aided Design, 44(2):132-142. [doi:10.1016/j.cad.2011.10.004]

Zou, Z., Morse, E.P., 2003. Applications of the gapspace model for multidimensional mechanical assemblies. Journal of Computing and Information Science in Engineering, 3(1):22-30. [doi:10.1115/1.1565072]

\section{中文概要}

题 目: 线性化行为模型的迭代统计公差分析

目 的: 分析由制造过程产生的变化对机构行为造成的影响。主 要分析线性化方法对失败率估计的影响, 从而平衡模 型线性化造成的误差、计算时间和结果准确性。

创新点: 简化优化步骤, 将机构行为线性化为几个部分, 取代整 体蒙特卡洛法, 并采用一种迭代算法得到更加精确的 结果。

方 法: 1. 采用带有非线性约束条件的几何线性化方法和基于 失败率置信区间的算法 (图3、4和5)；2. 以一个器 件连接器为例, 验证该算法在估计装配失败率上的作 用。

结 论: 1 . 线性化方法不影响蒙特卡洛仿真时间; 2 . 线性 化次数对计算时间和失败率估计准确率有很大影响 （表1）；3. 线性化迭代统计方法相对于蒙特卡洛法在 计算时间、计算精度和计算效率上有很大的优越性。

关键词: 公差分析; 失败率; 行为模型线性化; 蒙特卡洛线性化 